\title{
PERFIL EPIDEMIOLÓGICO E CLÍNICO DE IDOSOS HOSPITALIZADOS NO SETOR DE EMERGÊNCIA
}

Luiza Bruna Freire Sampaio ${ }^{1}$

Andréa Carvalho Araújo Moreira ${ }^{2}$

Francisco Eduardo Silva de Oliveira ${ }^{1}$

Iane Ximenes Teixeira ${ }^{2}$

Natália Frota Goyanna ${ }^{2}$

Vitória Lidia Pereira Sousa ${ }^{1}$

\author{
https://orcid.org/0000-0002-5628-508X \\ https://orcid.org/0000-0001-9855-1449 \\ https://orcid.org/0000-0002-9907-7715 \\ http://orcid.org/0000-0002-6790-6478 \\ https://orcid.org/0000-0002-8957-6828 \\ https://orcid.org/0000-0001-5004-3592
}

Objetivo: traçar o perfil epidemiológico e clínico de idosos atendidos em um serviço de emergência de um hospital de referência da zona norte do Estado do Ceará. Metodologia: Estudo transversal, realizado no período de julho a agosto de 2018, no setor de emergência de um hospital cearense. Participaram 80 idosos hospitalizados que responderam um formulário estruturado com dados epidemiológicos, clínicos e sobre os cuidados de enfermagem. Os resultados foram compilados com auxilio do Software R. Resultados: Prevaleceram idosos homens (51,25\%), diagnosticados por acidente vascular encefálico (38,75\%) e que tinham doenças crônicas associadas (65,00\%). O serviço de enfermagem foi avaliado como bom (62,5\%), mas os participantes sugeriram melhor agilidade (23,75\%) e garantia da privacidade (18,75\%). Conclusões: Idosos hospitalizados no setor da emergência possuem vulnerabilidades clínicas múltiplas, avaliam o serviço de enfermagem como bom, mas necessitam de melhor resolutividade em seu atendimento, bem como respeito ético.

Descritores: Enfermagem; Saúde do idoso; Hospitalização.

\section{EPIDEMIOLOGICALAND CLINICAL PROFILE OF HOSPITALIZED ELDERLY PEOPLE IN THE EMERGENCY SECTOR}

Objective: To outline the epidemiological and clinical profile of elderly people attended in an emergency service of a reference hospital in the northern zone of the State of Ceará. Methodology: Transversal study, accomplished from July to August of 2018 in the emergency sector of a hospital from Ceará. 80 hospitalized elderly people took part in the study and answered a structured form with data epidemiological, clinical and nursing care data. The results were compiled with the support of the Software R. Results: Men prevailed (51,25\%), diagnosed with cerebrovascular accident (38,75\%) and the associated chronic diseases (65,00\%). The nursing service was evaluated as good (62,5\%), but the participants proposed a better agility (23,75\%) and the assurance of privacy (18,75\%). Conclusion: Hospitalized elderly people in the emergency sector possess multiple clinical vulnerabilities, evaluated the nursing service as good, but required a better standard of problem-solving in the care, as well as ethical respect.

Descriptors: Nursing; Elderly health; Hospitalization.

\section{PERFIL EPIDEMIOLÓGICO Y CLÍNICO DE ANCIANOS INGRESADOS EN EL SECTOR DE URGENCIAS}

Objetivo: trazar el perfil epidemiológico y clínico de los ancianos atendidos en un servicio de urgencias de un hospital de referencia en la zona norte del Estado de Ceará. Metodología: Estudio transversal, realizado en el periodo de julio a agosto de 2018 en el sector de urgencias de un hospital cearense. Han participado 80 ancianos ingresados en el sector de urgencias que han respondido a un impreso estructurado con datos epidemiológicos, clínicos y bajo los cuidados de enfermería. Los resultados han sido allegados con el software R. Resultados: Han prevalecido ancianos hombres (51,25\%), diagnosticados con accidente vascular encefálico (el 38,75\%) y que poseían enfermedades crónicas asociadas (el 65\%). El servicio de enfermería ha sido evaluado como bueno (el 62,5\%), pero los participantes han sugerido mejor agilidad en el atendimiento (el 23,75\%) y garantía de la privacidad (el 18,75\%). Conclusiones: Ancianos ingresados en el sector de urgencias poseen vulnerabilidades clínicas múltiples, evalúan el servicio de enfermería como bueno, sin embargo necesitan mejor viveza en su atendimiento, tal como respeto ético.

Descriptores: Enfermería; Salud del anciano; Ingreso hospitalario.

IUniversidade Estadual Vale do Acaraú (UVA). E-mail: luizabrunal3@hotmail.com 2Universidade Federal do Ceará (UFC). E-mail: andreamoreiraueva@gmail.com 


\section{INTRODUÇÃO}

A transição demográfica é consequência da redução das taxas de mortalidade e natalidade e contribuiu fortemente para o fenômeno atual do envelhecimento populacional. Essa realidade tem sido observada nos países desenvolvidos e em desenvolvimento, o que requer acompanhamento, de acordo com as necessidades emergidas, haja vista que traz não apenas modificações na estrutura etária da população, mas aumento nos indices de morbidade, maior proporção de agravos e procedimentos médicos, além do aumento das doenças crônico-degenerativas com suas consequências indesejadas quando não tratadas adequadamente ${ }^{1-2}$

Nas unidades de emergências, é comum o atendimento ao idoso devido à maior frequência de problemas relacionados à idade, exigindo profissionais preparados para assistência imediata aos clientes. Portanto, é imprescindivel prezar pela manutenção da qualidade de vida dessa população, com vistas às possibilidades de prevenção de doenças, manutenção e reabilitação do seu estado de saúde ${ }^{3}$.

Vale ressaltar que, dentro do setor emergência, atender o idoso de forma integral é um grande desafio e, para isso, é necessário que os profissionais de saúde reconheçam suas necessidades peculiares em diferentes cenários de atuação ${ }^{4}$. Dentro do ambiente emergencial, os idosos são expostos a riscos, tais como declínio funcional, polifarmácia, infecções hospitalares e queda na qualidade de vida. Os problemas são exacerbados pelo prolongado tempo de permanência, mobilidade restrita e condições desconfortáveis, provocadas por um ambiente agitado, barulhento e pouco privativo ${ }^{5}$.

Essa situação leva, muitas vezes, a um quadro incapacitante, afetando a autonomia e funcionalidade dos idosos, gerando uma dificuldade para o desenvolvimento de atividades cotidianas, de forma independente, o que afeta de forma expressiva a qualidade de vida dessas pessoas ${ }^{2}$.

Investigar casos de pessoas idosas que procuram serviços de emergência numa situação de agravamento de saúde, e conhecer suas características socioeconômicas e de saúde poderá contribuir para identificar aspectos das condições de vida e da assistência em saúde neste nivel de atenção.

Diante disso, esse estudo objetivou traçar o perfil epidemiológico e clínico de idosos hospitalizados pelo serviço de emergência de um hospital de referência da zona norte do estado do Ceará.

\section{METODOLOGIA}

\section{Tipo do estudo}

Trata-se de um estudo transversal, do tipo descritivo com abordagem quantitativa.

\section{População e amostra}

A amostra não probabilística foi composta por 80 idosos internados ou em observação no setor de emergência, durante o período de julho e agosto de 2018.

Atendeu-se aos seguintes critérios de inclusão: idade mínima de 60 anos, conforme o critério estabelecido pela Organização Mundial da Saúde (OMS), que define como idoso o participante com 65 anos para os países desenvolvidos e 60 anos, para as pessoas de países subdesenvolvidos ou em desenvolvimento, como é o caso do Brasil. Idosos de ambos os sexos, com habilidade cognitiva autodeclarada e percebida, capazes de compreender e responder o instrumento.

Foram excluidos da amostra, devido a própria dinâmica do serviço, idosos em observação que evoluíram para alta hospitalar em poucas horas.

\section{Local de estudo}

A pesquisa ocorreu em um hospital universitário de grande porte, localizado na cidade de Sobral-CE, no setor de emergência.

O referido setor atende, prioritariamente, especialidades classificadas como alto risco. Além disso, atende demanda espontânea, sendo porta de entrada em traumatologia, neurologia, hematologia e nefrologia, assim como recebe transferência do Serviço de Atendimento Móvel de Urgência - SAMU, de hospitais de cidades vizinhas e encaminhamento de Unidades de Pronto Atendimento

\section{Coleta de dados}

Para coleta de dados, utilizou-se como instrumento de pesquisa um formulário estruturado com variáveis sociodemográficas (idade, procedência, sexo, escolaridade, estado civil, ocupação e renda), dados da hospitalização (motivo da hospitalização, categoria da hospitalização, dados clínicos, tempo de permanência, diagnósticos definidos ou hipotéticos, sinais e sintomas, exames realizados, presença de doenças crônicas e encaminhamentos) e cuidados de enfermagem (procedimentos de enfermagem e orientações de cuidados à saúde recebidos e sua frequência, avaliação e sugestões). Antes do início da pesquisa foi realizado um teste piloto com dez idosos. A coleta de dados ocorreu entre julho e agosto de 2018, em dias alternados.

\section{Procedimentos para análise de dados}

Para a análise das informações relacionadas ao perfil epidemiológico e clínico dos idosos hospitalizados no setor de emergência, os dados foram tabulados no software Excel (2013), analisados estatisticamente com o apoio do software R, versão 3.5.0 for Windows ${ }^{\circledR}$ e sintetizados em tabelas e gráficos.

Foi realizada uma análise descritiva dos dados que incluiu o cálculo de frequências absolutas, percentuais, e o intervalo de confiança. Para as proporções de variáveis categóricas foram calculados intervalos de confiança de $95 \%$. Para verificação de aderência à distribuição normal, foi aplicado o teste de Lilliefors, 
admitindo-se média, desvio padrão, mediana e o intervalo interquartílico das variáveis numéricas, em que os resultados de $\mathrm{p}<0,05$ foram sugeridos como nível de diferença significativa.

\section{Procedimentos éticos}

Esta pesquisa buscou seguir a Resolução 466/2012 do Conselho Nacional de Saúde ${ }^{(6)}$. Antes da coleta das informações, os participantes foram informados sobre os riscos e benefícios do estudo; em seguida, assinaram o Termo de Consentimento Livre e Esclarecido. A pesquisa foi submetida ao Comitê de Ética em Pesquisa (CEP) da Universidade Estadual Vale do Acaraú (UVA), sendo aprovado pelo parecer sob n으 2.681.950.

\section{RESULTADOS}

Neste estudo foram entrevistados 80 idosos internados ou em observação no setor de emergência de um hospital terciário. A tabela 1 demonstra os dados sociodemográfico dos idosos.

Tabela 1 - Distribuição dos idosos hospitalizados no setor de emergência de acordo com sexo, estado civil, ocupação, escolaridade e idade. Sobral-CE, 2018.

\begin{tabular}{|c|c|c|c|c|c|}
\hline Variável & & $\mathbf{N}$ & $\%$ & \multicolumn{2}{|c|}{ IC $95 \%$} \\
\hline \multicolumn{6}{|l|}{ 1. Sexo } \\
\hline Masculino & & 41 & 51,25 & 39,89 & 62,48 \\
\hline Feminino & & 39 & 48,75 & 37,51 & 60,10 \\
\hline \multicolumn{6}{|l|}{ 2. Estado Civil } \\
\hline Casado & & 47 & 58,75 & 47,18 & 69,46 \\
\hline Viúvo & & 28 & 35,00 & 24,89 & 46,55 \\
\hline Separado & & 4 & 5,00 & 01,61 & 12,99 \\
\hline Solteiro & & 1 & 1,25 & 0,065 & 07,72 \\
\hline \multicolumn{6}{|l|}{ 3. Ocupação } \\
\hline Aposentado & & 73 & 91,25 & 82,25 & 96,11 \\
\hline Trabalhador Rural & & 4 & 5,00 & 1,61 & 12,99 \\
\hline Dona de casa & & 3 & 3,75 & 0,97 & 11,31 \\
\hline \multicolumn{6}{|l|}{ 4. Escolaridade } \\
\hline Analfabeto & & 24 & 30,00 & 20,52 & 41,41 \\
\hline Ensino Fundamental incompleto & & 38 & 47,50 & 36,33 & 58,90 \\
\hline Ensino Fundamental Completo & & 6 & 7,50 & 3,08 & 16,19 \\
\hline Ensino Médio Incompleto & & 1 & 1,25 & 0,65 & 7,72 \\
\hline Ensino Médio completo & & 2 & 2,50 & 0,43 & 9,57 \\
\hline Ensino Superior completo & & 1 & 1,25 & 0,065 & 07,72 \\
\hline \multirow[t]{2}{*}{ Não souberam responder } & & 8 & 10,00 & 14,22 & 33,46 \\
\hline & Média & DP & Mediana & IIO & Valor $\mathrm{P}^{1}$ \\
\hline 5. Idade & 74,94 & 8,96 & 76,50 & 15,50 & 0,04 \\
\hline
\end{tabular}


Sobre as características clínicas dos idosos avaliados no estudo, os dados estão apresentados na tabela 2.

Tabela 2 - Distribuição de idosos de acordo com o tipo de hospitalização, unidade de internação, quantidade de hospitalizações, presença de doenças crônicas, exames realizados e tempo de permanência dos idosos hospitalizados no setor de emergência de um hospital terciário. Sobral-CE, 2018.

\begin{tabular}{|c|c|c|c|c|c|}
\hline Variável & & $\mathbf{N}$ & $\%$ & \multicolumn{2}{|c|}{ IC $95 \%$} \\
\hline \multicolumn{6}{|l|}{ 1. Tipo de Hospitalização } \\
\hline Internação & & 59 & 73,75 & 62,51 & 82,66 \\
\hline \multicolumn{6}{|c|}{ 3. Unidade de hospitalização } \\
\hline Baixa complexidade & & 49 & 61,25 & 49,66 & 71,74 \\
\hline Média Complexidade & & 14 & 17,50 & 10,23 & 27,95 \\
\hline Alta Complexidade & & 8 & 10,00 & 14,22 & 33,46 \\
\hline \multicolumn{6}{|c|}{ 4. Quantidade de hospitalizações } \\
\hline Uma vez & & 60 & 75,00 & 63,84 & 83,71 \\
\hline Duas vezes & & 19 & 23,75 & 15,24 & 34,81 \\
\hline Três vezes & & 1 & 1,250 & 0,065 & 07,72 \\
\hline \multicolumn{6}{|l|}{ 7. Exames Realizados } \\
\hline Laboratoriais & & 72 & 42,35 & 80,73 & 95,27 \\
\hline Tomografia & & 64 & 37,64 & 69,26 & 87,79 \\
\hline Raio-x & & 19 & 11,17 & 15,24 & 34,81 \\
\hline Ressonância & & 15 & 8,82 & 11,21 & 29,35 \\
\hline \multirow{2}{*}{ Tempo de Permanência e } & Média & DP & Mediana & IIO & Valor $\mathrm{P}^{1}$ \\
\hline & 6,22 & 8,50 & 3,00 & 7,00 & $<0,001$ \\
\hline
\end{tabular}


O gráfico 1 demonstra as causas de hospitalização de idosos por sexo. Verificou-se que o Acidente Vascular Encefálico (AVE) foi o principal motivo de hospitalização entre os idosos, correspondendo a $(38,75 \%)$ do total. Acrescenta-se que o percentual da ocorrência do AVE em mulheres foi quase o dobro quando comparados aos homens. Por outro lado, o índice de hospitalização por Insuficiência Renal, foi mais comum nos homens.

Gráfico 1 - Causas de hospitalização de idosos por sexo. Sobral-CE,2018.

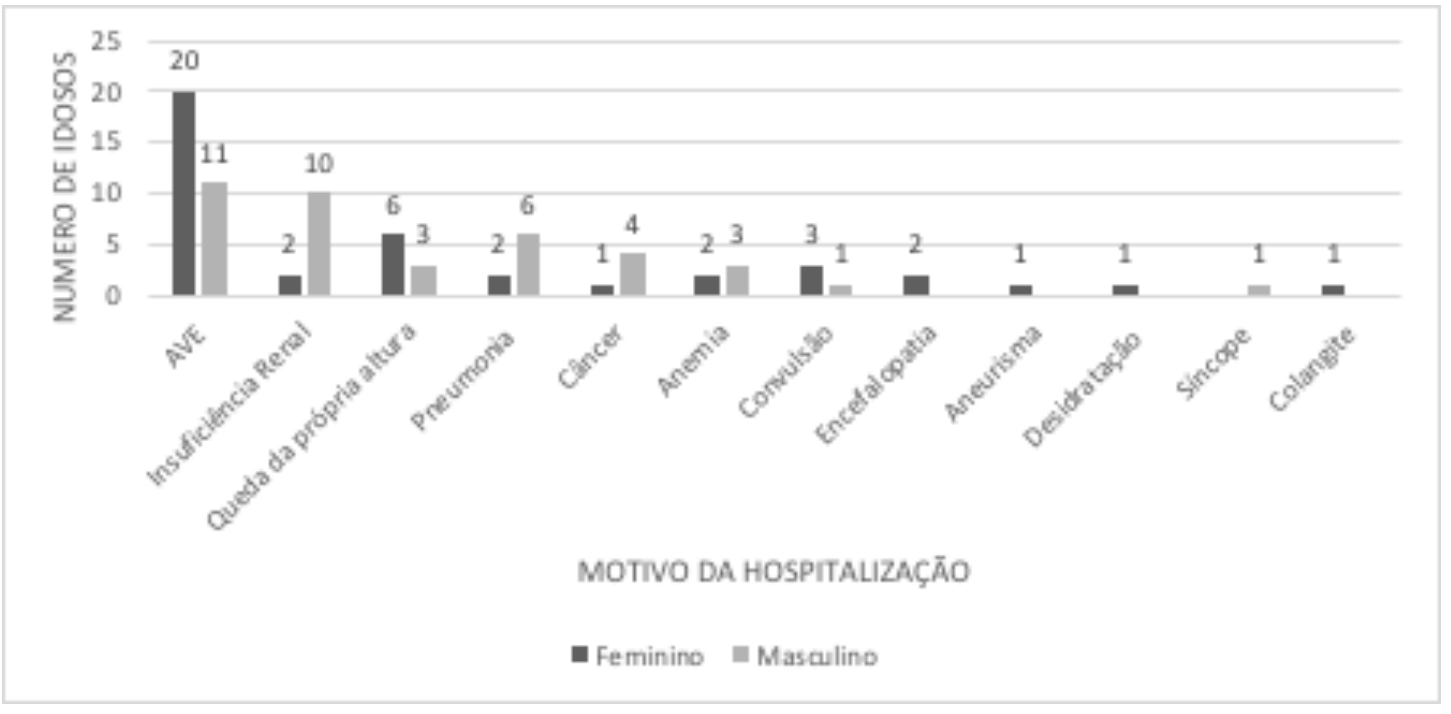

O gráfico 2 representa a distribuição de idosos quanto aos sinais e sintomas e procedimentos de enfermagem realizados no local da pesquisa. Nota-se que todos os idosos tiveram seus sinais vitais verificados, assim como o procedimento de punção venosa, porém quatro não precisaram de administração de medicamentos e necessitaram apenas de hidratação venosa. Os Cateterismos vesicais de demora e gástrico, representaram, respectivamente $(67,5 \%)$ e $(57,55 \%)$, realizados principalmente nos idosos admiti- dos para a alta complexidade. Ainda, destacaram entre os procedimentos de enfermagem, o banho no leito $(73,75 \%)$, mudança de decúbito (77,5\%), aspiração (40\%), gasometria (15\%), troca de curativo $(36,25 \%)$, auxílio nas refeições (65\%) e clister (1,25\%).

Os sinais e sintomas mais evidenciados foram o baixo nível de consciência (47,5\%), hemiparesia (31,25\%) e desvio de comissura labial (25\%), isto, justifica-se pelo motivo prevalente da hospitalização dos idosos ter sido o AVE.

Gráfico 2 - Distribuição de idosos quanto aos sinais esintomas e procedimentos de enfermagem realizadosno setor deemergência. Sobral-CE, 2018.

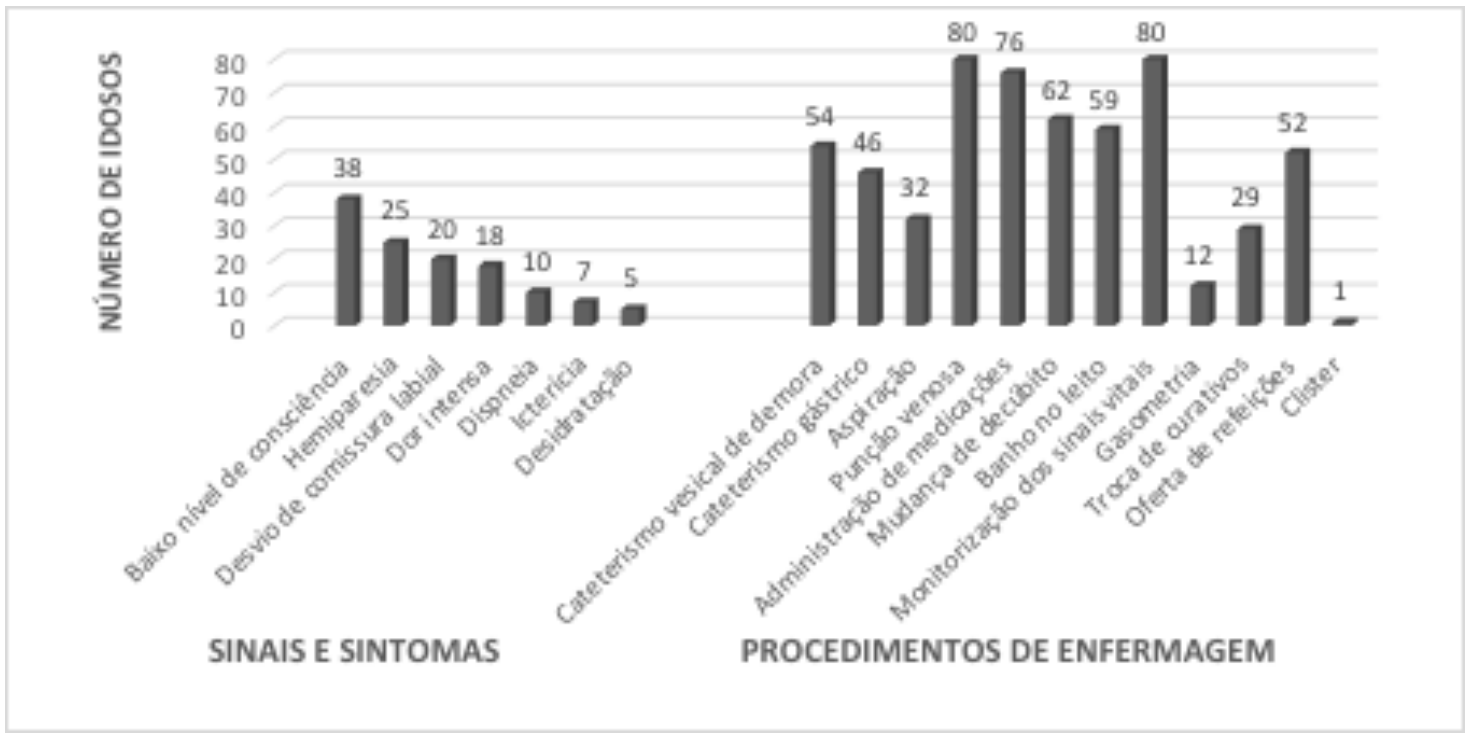


A tabela 3 demonstra as orientações recebidas pelos profissionais de enfermagem e as sugestões apontadas pelos participantes. Observou-se que mais da metade dos idosos e seus acompanhantes não tiveram orientações quanto ao estado de saúde, $(28,75 \%)$ re- lataram que só receberam orientações somente uma vez. Dos que informaram terem recebido orientações, apenas (47, 50\%) foram sobre condição de saúde, os demais foram orientações quanto a exames $(26,25 \%)$ e ou regras de setor $(22,5 \%)$.

Tabela 3 - Caracterização dos idosos quanto orientações recebidas, frequência de orientações, avaliações e sugestões relacionados aos cuidados de enfermagem. Sobral- CE, 2018.

\begin{tabular}{|c|c|c|c|c|}
\hline Variável & $\mathbf{N}$ & $\%$ & \multicolumn{2}{|c|}{ IC $95 \%$} \\
\hline $\begin{array}{l}\text { 1. Receberam orientações acerca dos cuida- } \\
\text { dos a saúde }\end{array}$ & 38 & 47,5 & 36,33 & 58,90 \\
\hline \multicolumn{5}{|l|}{ 2. Frequência das orientações } \\
\hline Diariamente & 4 & 5,000 & 1,61 & 12,99 \\
\hline Semanalmente & 11 & 13,75 & 7,39 & 23,69 \\
\hline Apenas uma única vez & 23 & 28,75 & 19,45 & 40,11 \\
\hline Não recebeu & 42 & 52,50 & 41,09 & 63,66 \\
\hline \multicolumn{5}{|l|}{ 3.Orientações Recebidas } \\
\hline Condição da saúde & 38 & 47,50 & 36,33 & 58,90 \\
\hline Exames & 21 & 26,25 & 17,33 & 37,48 \\
\hline Regras do Setor & 18 & 22,50 & 14,22 & 33,46 \\
\hline \multicolumn{5}{|l|}{ 3. Avaliação dos cuidados de enfermagem } \\
\hline Ótimo & 26 & 32,50 & 22,69 & 44,00 \\
\hline Bom & 50 & 62,50 & 50,92 & 72,86 \\
\hline Ruim & 4 & 5,000 & 01,61 & 12,99 \\
\hline \multicolumn{5}{|l|}{ 4. Sugestões de Melhoria } \\
\hline Agilidade no atendimento & 19 & 23,75 & 15,24 & 34,81 \\
\hline Melhoria na privacidade & 15 & 18,75 & 8,32 & 25,13 \\
\hline Melhora da estrutura física & 17 & 21,25 & 13,20 & 32,10 \\
\hline Ajuda para necessidades diárias dos idosos & 8 & 10,00 & 14,22 & 33,46 \\
\hline Ações de educação em saúde & 9 & 11,25 & 05,59 & 20,76 \\
\hline
\end{tabular}




\section{DISCUSSÃO}

Com relação ao perfil sociodemográfico dos idosos hospitalizados no setor da emergência, observou-se predomínio do sexo masculino, com faixa etária de 76 anos e seis meses, casados, aposentados e com baixa escolaridade. O predomínio do sexo masculino deve-se ao pouco envolvimento e cuidado do homem com sua saúde e por procurarem atendimento quando suas necessidades estão em estágios mais complexos, o que pode acarretar complicações e prejuízos em sua qualidade de vida ${ }^{7}$.

Os dados sociodemográficos encontrados foram semethantes em estudos nacionais ${ }^{8-9}$, e em estudo internacional ${ }^{10}$. No entanto, estudo nacional que analisou internações evitáveis pela atenção primária com idosos admitidos em pronto atendimento, no estado do Paraná, apontou prevalência no sexo feminino ${ }^{11}$.

Com relação ao tempo de hospitalização dos idosos, pelo menos metade permaneceu até três dias no setor da emergência. A permanência do usuário em periodo superior a 24 horas nos serviços de emergência, descaracteriza a assistência aos casos de emergência. Tal fato está associado à precariedade da rede assistencial, déficits na regulação dos leitos, falta de acesso à atenção ambulatorial especializada e à atenção hospitalar que, por sua vez, impactam o atendimento de emergência ${ }^{12}$.

O serviço de emergência tem como característica ser um ambiente dinâmico em que novos pacientes são admitidos continuamente. Com isso, os idosos que aguardam um leito de internação deixam de ser uma prioridade para o enfermeiro, que direciona maior atenção aos que aguardam por atendimento e apresentam risco de morte ${ }^{13}$.

Além disso, o tempo prolongando de internação agrava o estado de saúde do idoso, tornando-o mais propenso a desenvolver infecções hospitalares, lesões por pressão, assim como comprometimento psicológico, devido às características inerentes aos serviços hospitalares. A internação também afeta os domínios de autonomia e independência do idoso, pelo fato dele estar em ambiente não familiar, exposto a rotinas diferentes, com limitação da execução de atividades diárias, restrição ao leito, alteração do sono, iatrogenia, má nutrição e delirium ${ }^{14}$.

Dessa forma, faz-se necessário a efetividade da atenção primária nas ações de promoção à saúde voltadas a esse grupo etário, considerando a prevalência de multicomorbidade, cujas intervenções são fundamentais para prevenir o agravamento dos casos e mesmo a hospitalização.

Neste estudo, mais da metade dos idosos relataram tratamento para as doenças crônicas hipertensão arterial sistêmica e diabetes mellitus. Estudos nacionais realizados nessa população reportam resultados similares, onde evidenciou-se que as comorbidades mais frequentes em idosos hospitalizados foram as mesmas ${ }^{15}$. Na perspectiva internacional, foi possivel identificar resultados análogos aos achados nacionais $^{16}$. A presença de doenças crônicas e comorbidades associadas à vida mais dinâmica do idoso contemporâneo favorece a ocorrência de quedas, resultando em significativas consequências psicológicas, físicas e sociais para a vida desses indivíduos ${ }^{11}$.

As quedas da própria altura também ficaram em evidência neste estudo. A queda de pessoas idosas é uma causa crescente de lesões, custos de tratamento e morte. Além disso, mais da metade dos idosos que já tiveram um episódio de queda desenvolvem o medo de cair novamente com consequente inatividade física, piora do equilibrio e aumentando o risco de novas quedas ${ }^{17-18}$

Quanto aos sinais e sintomas apresentados, em sua maioria, são compatíveis com o AVE, sendo o diagnóstico responsável pela principal causa de hospitalização. Corroborando com os resultados deste estudo, uma pesquisa desenvolvida na Paraiba, evidenciou achados semelhantes ${ }^{(19)}$

Sobre os procedimentos de enfermagem, foi identificado que os mais realizados foram: cateterismo vesical e gástrico em idosos hospitalizados. Os dados são semelhantes a uma pesquisa transversal, realizada em um hospital no estado do Piauí ${ }^{20}$. Sabe-se que os procedimentos invasivos contribuem para tempo maior de internação prolongada no ambiente hospitalar, esses procedimentos são vitais no prolongamento da vida, no entanto, os mesmos são comumente relacionados ao risco de complicações clínicas ${ }^{20}$

Neste estudo, o serviço de enfermagem foi avaliado como bom, onde os profissionais preocupam-se em ajudar os idosos a aliviar dores e desconforto. Os achados colaboram com uma revisão integrativa, onde foi evidenciado que a simpatia, respeito e compartilhamento de informações ao realizar um procedimento de enfermagem, são medidas que proporcionam conforto, contribuindo para a satisfação dos idosos atendidos pelo serviço de enfermagem no setor de emergência, minimizando sinais e sintomas dos idosos ${ }^{21}$.

Apesar do serviço de enfermagem ter sido avaliado positivamente, foram apontadas pelos participantes as seguintes sugestões: agilidade no atendimento, melhoria na privacidade, melhora da estrutura física, ajuda para necessidades diárias do idoso e ações de educação em saúde. Estudo realizado na Espanha revelou que as características do ambiente como superlotação de pacientes aguardando atendimento, número insuficiente de profissionais e falta de espaço físico pode influenciar na privacidade do paciente e, consequentemente, no cuidado realizado 22 
A manutenção da privacidade é muito importante, pois no setor de emergência, os espaços são, frequentemente, superlotados e compartilhados entre os usuários. Para os idosos, a falta de privacidade, somada ao ambiente agitado, pode levar à desorientação, sensação de incapacidade e perda da autonomia ${ }^{23}$. Dessa forma, quando o enfermeiro não garante a privacidade do paciente, ele comete uma má conduta ética, visto que o código de ética dos profissionais de enfermagem - Resolução COFEN no 564/2017 - prevê uma série de recomendações sobre os princípios fundamentais do exercício de enfermagem, sendo enfático no respeito, autonomia, pudor, privacidade e a intimidade do ser humano em todo seu ciclo vital ${ }^{24}$.

\section{Limitação do estudo}

Destacam-se como limitação deste estudo, a coleta de dados ter sido realizada em um único hospital, considerando o curto período de tempo. Vale ressaltar que houve perdas da amostra devido à própria dinâmica do serviço, como os idosos em observação que evoluíram para alta hospitalar em poucas horas.

\section{Contribuições para a prática}

Os resultados do estudo podem servir de base para outros estudos e reflexões acerca da temática, além de fornecer subsídios para o planejamento, implementação e avaliação de ações e políticas de saúde pública, visando um melhor acolhi- mento e uma melhor atenção ao idoso hospitalizado no setor de emergência.

\section{CONCLUSÃO}

Os resultados deste estudo reiteram a importância de levantar informações em relação ao perfil clínico e epidemiológico de idosos hospitalizados no setor de emergência.

Ressalta-se a importância de melhorar a assistência prestada ao idoso no serviço de emergência, uma vez que os idosos já chegam com um estado de saúde vulnerável. Além disso, apesar da maioria dos idosos avaliarem o serviço de enfermagem como bom, apontaram como sugestões, a agilidade no atendimento e a garantia da privacidade.

A falta de zelo dos profissionais de enfermagem em não garantir a privacidade do paciente retrata uma realidade preocupante em que não são respeitados os princípios éticos elementares no cuidado humano, fato esse que pode implicar em danos legais aos profissionais e ao serviço.

Contribuições dos Autores: Concepção, desenho, análise, interpretação dos dados, redação do artigo, revisão crítica e revisão final: Luiza Bruna Freire Sampaio, Andréa Carvalho Araújo Moreira, Francisco Eduardo Silva de Oliveira, Iane Ximenes Teixeira, Natália Frota Goyanna, Vitória Lídia Pereira Sousa.

\section{REFERÊNCIAS}

1.Melo LA, Ferreira LMBM, Santos MM, Lima KC. Fatores socioeconômicos, demográficos e regionais associados ao envelhecimento populacional. Rev. bras. geriatr. gerontol. [internet]. 2017 [acesso em 20 nov 2019]: 20(4). Disponivel em: http://www.scielo.br/pdf/rbgg/ v20n4/pt_1981-2256-rbgg-20-04-00493.pdf.

2. Costa NRCD, Aguiar MIF, Rolim ILTP, Rabelo PPC, Oliveira DLA, Barbosa YC. Política de saúde do idoso: percepção dos profissionais sobre sua implementação na atenção básica/Health policy for elderly people: perception of professionals about its implementation in primary care. Rev Pesq. Saúde. [internet].2016 [acesso em 20 nov 2019]: 16(2). Disponivel em: http://www. periodicoseletronicos.ufma. br/index.php/revistahuufma/article/view/4239.

3.Coutinho MLN, Samúdio MA. Andrade LM, Coutinho RN. Silva DMA. Perfil sociodemográfico e processo de hospitalização de idosos atendidos em um hospital de emergências. Rev Rene (Online). 2015 [acesso em 17 nov 2019]: 6(6). Disponivel em: http://repositorio.ufc. br/ri/bitstream/riufc/14763/1/2015_art_mlncoutinho.pdf

4. Teixeira JJM, Bastos GCFC, Souza ACL. Perfil de internação de idosos. Rev. Soc. Bras. Clin. Méd. [internet].2017 [acesso em 17 nov 2019]: 15(1). Disponivel em: http://docs.bvsalud.org/biblioref/2017/04/833048/15-20.pdf

5. Ministério da Saúde (BR). Secretaria de Atenção à Saúde. Departamento de Atenção Básica. Envelhecimento e saúde da pessoa idosa. Cadernos de Atenção Básica no 19; 2006.

6. Ministério da Saúde (BR). Conselho Nacional de Saúde, Comissão Nacional de ética em Pesquisa. Resolução n 466 de 12 de dezembro de 2012: aprova as diretrizes e normas regulamentadoras de pesquisa envolvendo seres Humanos; 2012

7. Nascimento AAS, Cunha BSS, Sá SPC. Perfil clínico e sociodemografico de internação de idosos na unidade de emergência de um hospital geral. Estud. interdiscip. envelhec. [internet]. 2014 [acesso em 17 nov 2019]:19(1). Disponivel em: http://www.seer.ufrgs.br/index.php/RevEnvelhecer/article/view/20963/31009. 
8. Oliveira C, Santos I, Rocca A, Dobri Nascimento G. Perfil epidemiológico de pacientes idosos atendidos em um pronto-socorro de hospital universitário brasileiro. Rev. med. (São Paulo). [internet]. 2018 [acesso em 10 out 2019]: 97 (1). Disponivel em: http://www.revistas.usp.br/revistadc/article/view/141366.

9.Lopes MCBT, Lage JSS, Vancini-Campanharo CR, Okuno MFP, Batista REA. Fatores associados ao comprometimento funcional de idosos internados no serviço de emergência. Einstein (São Paulo). [Internet]. 2015 [acesso em 15 out 2019]: 13(2). Disponivel em:http://www. scielo.br/scielo.php?script=sci_arttextEpid=S1679-45082015000200007\&lng=en. http://dx.doi.org/10.1590/S1679-45082015AO3327.

10. Karaveli A, Cerit GN, Özyurt E. Evaluation of admission causes and mortality rates of 65 years of age and older patientes admitted from the emergency department to the intensive care unit. Turkish jornal of geriatrics. [internet]. 2018 [acesso em 19 nov 2019]:21 (4). Disponivel em: http://geriatri.dergisi.org/abstract.php?id=1076.

11. Rissardo LK, Rego AS, Scolari GAS, Radovanovic CAT, Decesaro MN, Carreira L. Idosos atendidos em unidade de pronto-atendimento por condições sensiveis à atenção primária à saúde. REME rev. min. enferm. [internet]. 2016 [acesso em 15 out 2019 ]: Disponivel em. https://www.reme.org.br/artigo/detalhes/1107.

12. Nascimento ERP, Silva SG, Souza BC, Souza DD, Germer Netto A. Ambiência de uma emergência hospitalar para o cuidado ao idoso: percepção dos profissionais de enfermagem. Esc Anna Nery Rev. Enferm. [internet]. 2015 [acesso em 17 out 2019]: 19(2). Disponivel em: http://www.scielo.br/pdf/ean/v19n2/1414-8145-ean-19-02-0338.pdf.

13. Andrade LAS, Santos SP, Corpolato RC. Cuidado do idoso no setor de emergência: uma revisão integrativa. Rev. bras. geriatr. gerontol. [internet]. 2018 [acesso em 19 nov]: 21(2). Disponivel em: http://www.scielo.br/pdf/rbgg/v2ln2/pt_1809-9823-rbgg-21-02-00243. pdf.

14. Freitas SLS, Santos ELS, Pereira LSM, Lustosa LP. Vulnerabilidade dos idosos na alta hospitalar. Vulnerabilidade fisica de idosos na alta hospitalar. Fisioter pesqui. [internet]. 2017 [acesso em 13 out 2019]:24(3). Disponivel em: http://www.scielo.br/pdf/fp/v24n3/23169117-fp-24-03-00253.pdf

15. Campos MOC, Rodrigues Neto EM, Cunha JCL, Andrade LM, Fonteles MMF, Bezerra JCP. Condições de saúde fisica e mental dos idosos atendidos em urgência e emergência. Rev. enferm. UFPE on line. [internet]. 2017: 11(10). [acesso em 17 nov 2019]. DOI: 10.5205/ reuol.10712-95194-3-SM.1110sup20172.

16. Miró Ò, Aguilo S, Jacob J, Puente PH, Jiménes VC, Brizzi Bn et al. Profile of older patients attended in the emergency department after falls: A FALL-ER registry study of the magnitude of the problem and opportunities for improving hospital emergency care. Emergencias. [internet]. 2018 [acesso em 19 nov 2019]: 30(4). Disponivel em: www.researchgate.net/publication/326802904_Profile_of_ older_patients_attended_in_the_emergency_department_after_falls_A_FALLER_registry_study_of_the_magnitude_of_the_problem_and_opportunities_for_improving_hospital_emergency_care/citation/download

17. Freitas MG, Bonolo PF, Moraes EN, Machado CJ. Idosos atendidos em serviços de urgência no Brasil: um estudo para vítimas de quedas e de acidentes de trânsito. Ciênc. saúde coletiva (Online) [Internet]. 2015 [acesso 21 nov 2019]:20(3). Disponivel em: http://www. scielo.br/pdf/csc/v20n3/pt_1413-8123-csc-20-03-00701.pdf.

18. Sung YH, Cho MS, Kwon IG, Jung YY, Song MR, Kim K, et al. Evaluation of falls by inpatients in an acute care hospital in Korea using the Morse Fall Scale. Inter Journal of Nursing Practice. [internet]. 2014 [acesso em 30 nov 2019]: 20(5). Disponivel em: https://www. ncbi.nlm.nih.gov/pubmed/24118515.

19. Carvalho MA, Coutinho APO, Carvalho GDA, Queiróz DTG, Santos SR. Epidemiologia dos acidentes vasculares encefálicos atendidos por meio do serviço de atendimento móvel de urgência. Rev. enferm. UFPE on line. [internet]. 2015 [acesso em 14 nov 2019]:9(3). DOI: 10.5205/reuol.7505-65182-1-RV.0903201501.

20. Sprung CL, Artigas A, Kesecioglu J, Pezzi A, Wiis J, Pirracchio R, et al. The Eldicus prospective, observational study of triage decision making in European intensive care units. Part II: intensive care benefit for the elderly. Crit Care Med [Internet]. 2012 [acesso em 29 nov 2019]:40(1). Disponivel: https://www.ncbi.nlm.nih.gov/pubmed/22001580.

21. Acosta AM, Marques GQ, Levandovski PF, Peralta JP, Lima MADS. Satisfação dos usuários com cuidados de enfermagem em serviço de emergência: uma revisão integrativa. REME rev min. enferm. [internet]. 2016 [acesso 22 nov 2019]: Disponivel

22. Grossmann FF, Zumbrunn T, Ciprian S. Stephan FP. Woy N. Bingisser R, et al. Undertriage in older emergency department patients: tilting against windmills? PLos ONE. [Internet] .2014 [acesso em 23 nov. 2019]: 9(8). Disponivel em: http:// journals.plos.org/plosone/ article?id=10.1371/journal. pone.0106203).

23. Santos MT, Lima MADS, Zucatti PB. Elder-friendly emergency services in Brazil: necessary conditions for care. Rev. Esc. Enferm. USP. [internet]. 2016 [acesso em 30 nov 2019]:50(4). DOI: http://dx.doi.org/10.1590/S0080-623420160000500008.

24. Conselho Federal de Enfermagem (Cofen). Resolução n. 564 de 06 nov.2017. Dispõe sobre a aprovação da reformulação do código de ética dos profissionais de enfermagem, Brasília; 2017. 